\title{
Wildbeuter der Steinzeit Die ersten Siedler im Wurzener Land
}

\author{
Volkmar Geupel
}

Dem Andenken an Frida und Reinhold Birke

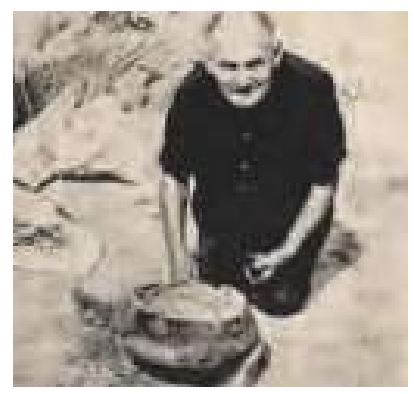

Reinhold Birke (1896-1973), um 1960
Unser Wissen über die früheste Besiedelung des Wurzener Raumes basiert in einem hohen Maße auf Sammelfunden, die nahezu allein den Geländebegehungen des Wurzener Heimatforschers Reinhold Birke (1896-1973) verdankt werden. Von der zweiten Hälfte der 1920er Jahre bis Ende 1966, als die "Sammlung Birke" geschlossen in das seinerzeitige Landesmuseum für Vorgeschichte Dresden gelangte, hat R. Birke unermüdlich zahllose Feldfluren, durch Steinbrüche und Sandgruben verursachte Bodenaufschlüsse, aber auch siedlungsgünstig erscheinende Geländepunkte im weiteren Umkreis von Wurzen nach prähistorischen Oberflächenfunden abgesucht. Die dabei erkannten Fundstellen, die den großen Zeitraum von der Altsteinzeit bis zum Mittelalter umfassen, wurden fortan regelmäßig von ihm begangen, das Fundgut beschriftet und dem Landesmuseum als der seinerzeit für die Archäologische Denkmalpflege in Sachsen zuständigen Institution angezeigt. Letzteres besorgte vor allem die Ehefrau Frida (19001982), wie die Fülle von Fundmeldungen und eine umfangreiche Korrespondenz in den betreffenden Ortsakten des heutigen Landesamtes für Archäologie bezeugen. Vor allem waren es steinzeitliche Werkzeuge, deren Herstellung und Verwendung vor Jahrtausenden den Schlosser R. Birke als Handwerker interessierten. Sein autodidaktisch erworbenes Wissen vertiefte er, oft von seiner Frau begleitet, durch die Teilnahme an den jährlichen Schulungen für die ehrenamtlichen Kreispfleger für Bodenaltertümer des Landesmuseums und an den Tagungen des Fachausschusses Ur- und Frühgeschichte im damaligen Kulturbund der DDR. In vierzig Jahren akribischer Geländetätigkeit erschloss R. Birke rund um seine Heimatstadt Wurzen ein Fundgebiet, in welchem sich auf den Kuppen und Talkanten beiderseits der Mulde die ehemaligen Siedlungsplätze aus der späten Alt- und der Mittelsteinzeit »wie Perlen auf einer Schnur« reihen. Muldeabwärts entdeckte er 1932 den Kapellenberg in Groitzsch bei Eilenburg als jungpaläolithischen Fundplatz, über dessen Auffindung er 1954 in einem vom seinerzeitigen Landesarchäologen W. Coblenz angeregten Aufsatz berichtete. ${ }^{1}$ Seit den Ausgrabungen H. Hanitzschs von 1954 bis 1961 gilt der Groitzscher Kapellenberg als überregional bedeutende Freilandstation des mitteldeutschen Spätmagdaléniens. ${ }^{2}$

1966 habe ich das gesamte steinzeitliche Fundmaterial aufgearbeitet ${ }^{3}$ und für die Überführung in das Landesmuseum vorbereitet. Dabei konnte ich mich von der hohen Präzision, mit der Birkes die Fundstellen im Gelände fixiert, über Jahrzehnte überwacht und die gesammelten Artefakte bezeichnet haben, überzeugen. Gern und dankbar erinnere ich mich an die Gastfreundschaft in ihrer Wohnung in Wurzen, an die Exkursionen zu ihren Fundstellen und die vielen, stets bereitwillig erteilten Auskünfte. So ist das im Folgenden gezeichnete Bild von der ältesten Besiedlung des Umlandes von Wurzen gewissermaßen in den Fußspuren R. Birkes entstanden, ohne dessen zahllose Flurbegehungen unser Wissen hierüber sehr viel lückenhafter wäre.

Das früheste Zeugnis für die Anwesenheit des urgeschichtlichen Menschen im Wurzener Muldeland stammt nach formenkundlichem Vergleich (s. unten) aus der ersten Hälfte der letzten der drei norddeutschen Eiszeiten, der Weichseleiszeit (um 80.0000 - um 9.600 v. Chr. ${ }^{4}$ ), die mit ihrer Eisdecke Sachsen nicht mehr erreicht hatte, deren Inlandeis vielmehr im Zuge von Fläming und Lausitzer Grenzwall liegen blieb. ${ }^{5}$ Es handelt sich um ein Artefaktensemble aus Feuerstein vom Spitzberg bei Lüptitz ${ }^{6}$, einem ehemals steilen Felskegel aus Quarzporphyr ${ }^{7}$, der vor seinem Abbau durch Steinbrüche die Umgebung um etwa $60 \mathrm{~m}$ überragt hatte ${ }^{8}$ und eine der auffallenden Erhebungen des Dornreichenbach-Hohburger Porphyrhügellandes, Teil des Nordsächsischen Platten- und Hügellandes ${ }^{9}$, bildete. Das Fundinventar besteht aus mehreren präparierten Kernsteinen, aus Abschlägen von solchen mit der typischen facettierten Schlagbasis und einigen fertigen Geräten wie das beidflächig flächenretuschierte Keilmesser - ein im Querschnitt keilförmiges Werkzeug mit einer 
scharfen und einer stumpfen Längskante - und der Fäustel. Steinschlagtechnik und Gerättypen weisen in das Mittelpaläolithikum (Mittlere Altsteinzeit), wobei Übereinstimmungen am ehesten mit den wahrscheinlich aus der ersten Hälfte der Weichseleiszeit stammenden Steingerätfunden vom Petersberg bei Halle (Saale) $)^{10}$ zu bestehen scheinen, die auf Grund ihres geologischen Alters dem späten Mittelpaläolithikum zugeordnet werden. Wie ehemals der Lüptitzer Spitzberg bildet auch der Petersberg eine beherrschende Bergkuppe mit hervorragender Fernsicht. Möglicherweise steht dahinter ein bestimmtes jagdtechnisches Verhalten der diese markanten Berge als Lagerplätze nutzenden altsteinzeitlichen Jäger, die $\mathrm{zu}$ dem Menschentyp des Neandertalers (Homo neanderthalensis) gehörten. Ihre Jagdbeute fanden sie unter der kaltzeitlichen Tierwelt wie Mammut, Fellnashorn, Moschusochse, Rentier, Höhlenbär und Höhlenlöwe. ${ }^{11}$

Der zeitlich nächste Fundniederschlag im Wurzener Land gehört bereits in die Spätphase der Weichseleiszeit. Nach der geologisch-klimatischen Gliederung handelt es sich um die rund 2.200 Jahre (um 11.800 - um 9.600 v. Chr. ${ }^{12}$ ) umfassende Zeitspanne der Dryasoder Tundrazeit, welche in die drei Abschnitte Dryas I (= Älteste), II (= Ältere) und III (= Jüngere Dryaszeit) und die diese trennenden Wärmeschwankungen Bölling- und Alleröd-Interstadial unterteilt wird. Während dieses Zeitraumes, mindestens aber bis an den Beginn der Jüngeren Dryaszeit, hielten sich in Mitteldeutschland Bevölkerungsgruppen auf, deren archäologische Kultur nach der französischen Höhle ,Grotte de la Madeleine in Tursac (Dordogne) als Magdalénien bezeichnet wird ${ }^{13}$ und die jüngste Kulturstufe des Jungpaläolithikums (Jüngere Altsteinzeit) ist. Das Jungpaläolithikum begann um 40.000 v. Chr. und war vom modernen, heutigen Menschen (Homo sapiens) getragen. Im Magdalénien wurde der Höhepunkt der eiszeitlichen Kunst erreicht weltberühmt sind die im mittleren und jüngeren Magdalénien polychrom ausgemalten »Bilderhöhlen« wie z. B. in Lascaux (Dordogne, Frankreich) oder in Altamira (Santander, Spanien). In dem Fundmaterial Mitteldeutschlands, das ausschließlich in die Spätphase dieser Kultur gehört, zeugen davon auf Steinplatten, Geweih und Knochen gravierte naturalistische Tierdarstellungen, vornehmlich des Hauptjagdtieres Wildpferd, ferner kommen aus Elfenbein und Geweih geschnittene Statuetten schematisierter Menschen (Frauen) vor. Von dem mehrphasigen Fundplatz auf dem Kapellenberg in Groitzsch bei Eilenburg stammt

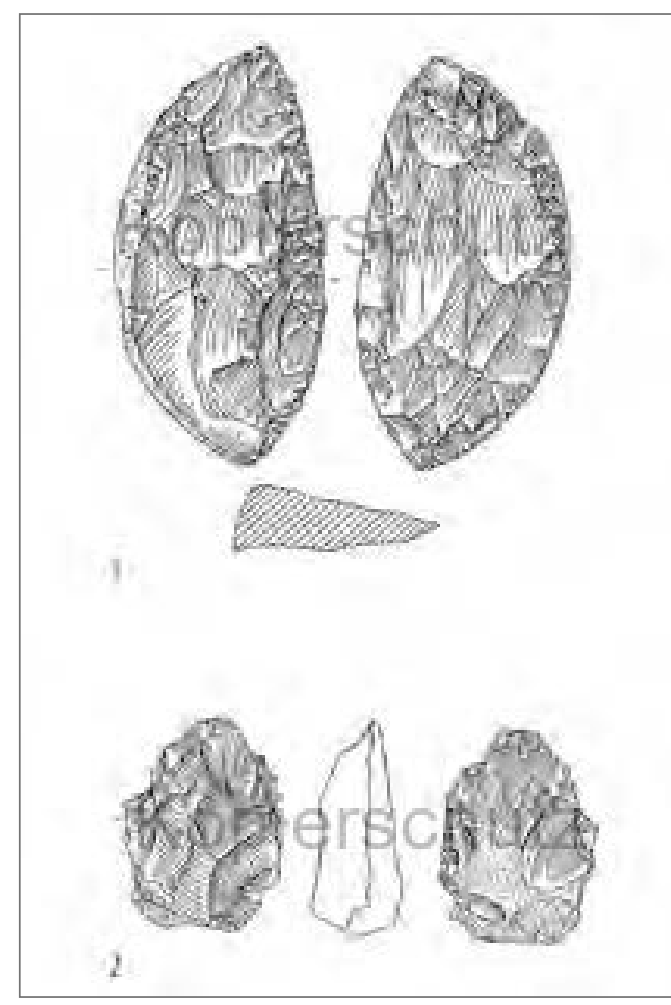

eine 1958 bei den Ausgrabungen H. Hanitzschs entdeckte kleine Schieferplatte mit der Einritzung von drei Wildpferdköpfen, an deren Hälsen tiefe, als "magische Verwundung " gedeutete Einstiche angebracht waren. ${ }^{14}$ Im Wurzener Muldeland konnte R. Birke auf dem Wachtelberg in Dehnitz mit einer Reihe kulturspezifischer Artefakte wie Bohrer und Rückenmesser einen weiteren, aber kleineren Rastplatz aus der Zeit des Spätmagdaléniens nachweisen. ${ }^{15}$ Neben Bohrern und Rückenmesserchen gehör-
Lüptitz, Spitzberg: Keilmesser (1) und Fäustel (2). 2:3.

(aus: V. Geupel 1972 [Anm. 6], Abb. 3, 1, 3). ( ) Landesamt für Archäologie Sachsen

Dehnitz, Wachtelberg: Bohrer (1-2), Rückenmesser (3-4), Klingenkratzer (5), Stichel (6) und Kombinationsgerät

(Stichel/Kratzer) (7). 2:3.

(aus: V. Geupel 1967 [Anm. 15], Abb. 2,$1 ; 3,5 ; 4,1 ; 5,3,4,9 ; 6,4)$. () Landesamt für Archäologie Sachsen

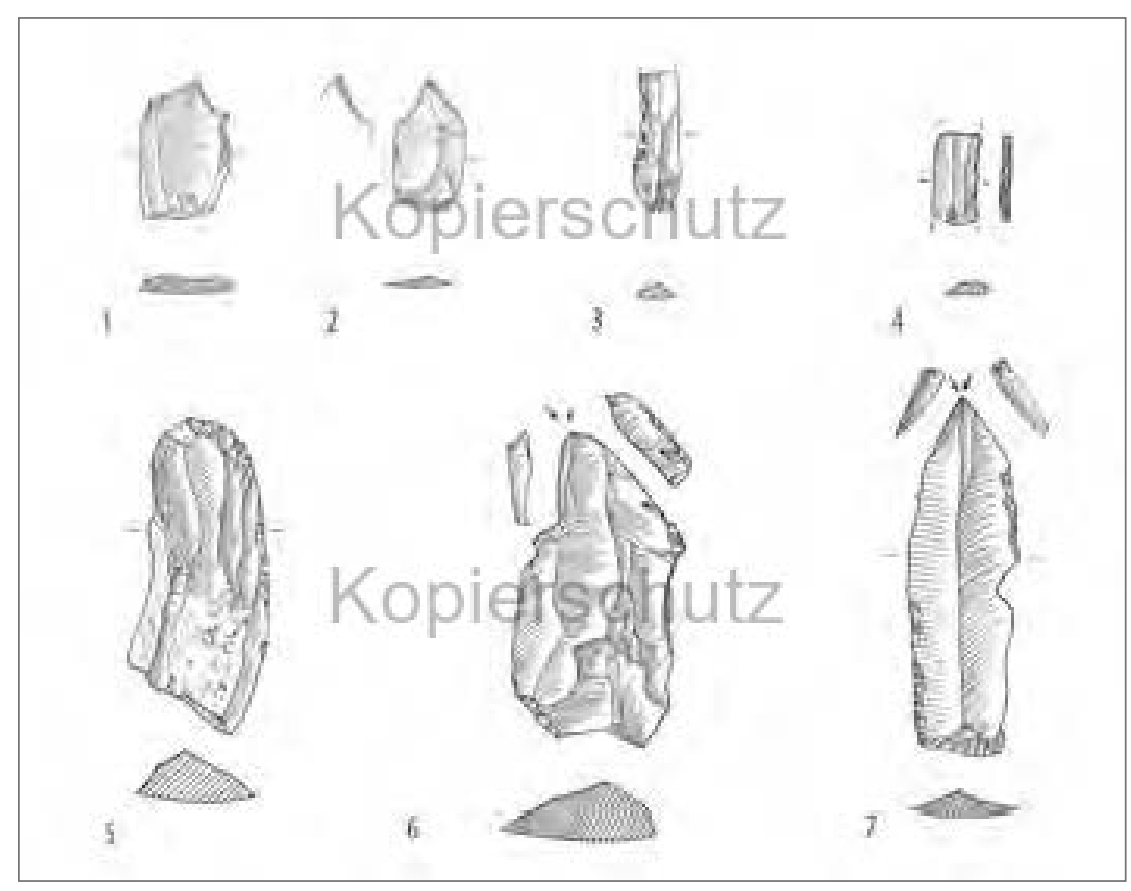


ten Klingenkratzer und zahlreiche Stichel sowie in der Endphase Rückenspitzen zum allgemeinen Werkzeugbestand des hiesigen Spätmagdaléniens. Auf Grund von einander ausschließenden Leittypen und Unterschieden im Artefaktbestand geben sich mehrere zeitlich und regional verschiedene Formengruppen $\mathrm{zu}$ erkennen. ${ }^{16}$ Ausgangsprodukte für die meisten Feuersteingeräte waren gestreckte bis langschmale Abschläge mit parallelen Kanten (Klingen), die von speziell präparierten Kernsteinen geschlagen worden waren. ${ }^{17}$ Diese den Beginn des Jungpaläolithikums (s. oben) markierende Steinbearbeitungstechnik erlaubte die rasche Herstellung größerer Serien von Klingen und führte zu einer Standardisierung der Geräte. Von den unter den natürlichen Bedingungen der ausklingenden Eiszeit lebenden Tieren war das Wildpferd die wichtigste Jagdbeute des Menschen (welches wohl auch deshalb in der künstlerischen Wiedergabe - wie $\mathrm{z}$. B. in Groitzsch - häufig erscheint), gefolgt vom Rentier. $^{18}$

Im späten Alleröd-Interstadial ${ }^{19}$ und im Übergang zur Jüngeren Dryaszeit breiteten sich die
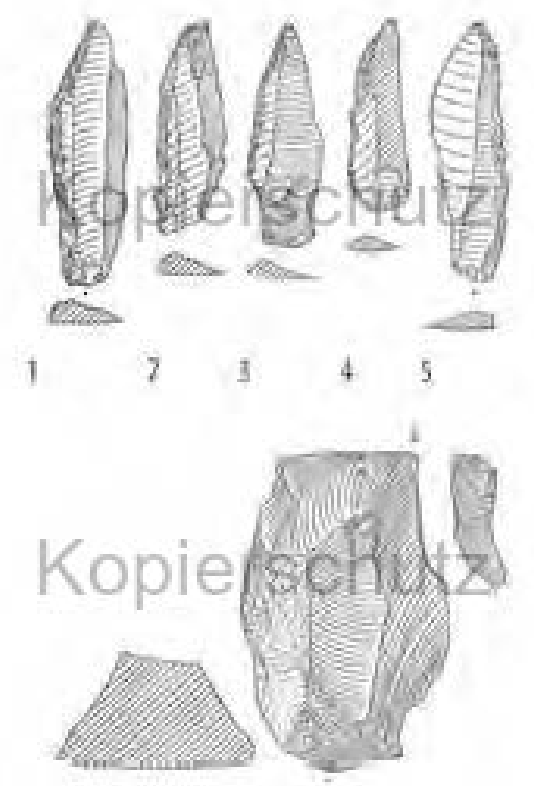

6

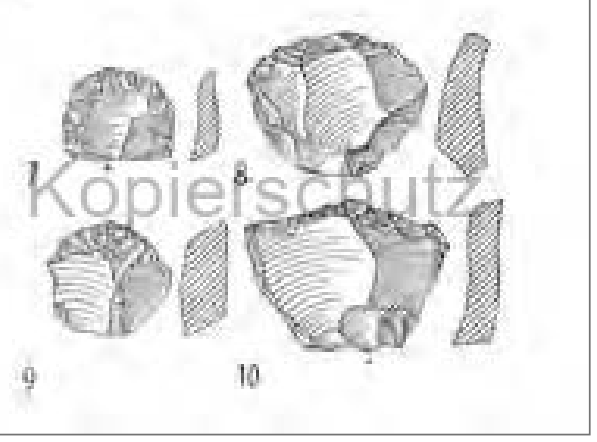

Rückenspitzen- oder »Federmesser«-Gruppen aus $^{20}$, für welche asymmetrische Rückenspitzen - Spitzen mit einer scharfen und einer gebogenen oder geknickten und durch Retuschierung gestumpften Längskante $(» \text { Rücken })^{21}$ - namengebend waren. Sie stellten eine weiträumige Erscheinung mit Entsprechungen im östlichen und südlichen Mitteleuropa sowie in Südwesteuropa dar. ${ }^{22}$ Fundstellen, in deren Steingeräteinventar Rückenspitzen vorkommen, entdeckte R. Birke in Dehnitz (2 Plätze), Oelschütz, Pausitz, Schmölen, Walzig und Zöhda (2 Plätze). ${ }^{23}$ Von diesen ist der Fundplatz »Wüste Kirche« bei Dehnitz mit sieben Rückenspitzen der materialreichste, mehrheitlich handelt es sich aber um kleine oder mit mittelsteinzeitlichen Geräten vermischte Fundkomplexe, die nur eine oder zwei Rückenspitzen enthalten. Der Werkzeugbestand war im Vergleich mit dem klassischen Magdalénien typenarm und beschränkte sich im Wesentlichen auf Rückenspitzen als Pfeilbewehrungen, kurze Kratzer und meist grob gearbeitete Stichel. Die nordwestsächsischen Funde lassen sich am ehesten der Rissener Gruppe des Rückenspitzen-Kreises zuordnen, die in dem an Sachsen nördlich angrenzenden Tiefland des Mittelelbgebietes und Brandenburgs die wichtigste Federmesser-Gruppe darstellte. ${ }^{24}$ Sie war aus dem Spätmagdalénien hervorgegangen und hatte die weiteste geographische Verbreitung mit mehreren lokalen Ausprägungen. ${ }^{25}$ So erscheinen in deren südöstlichem Verbreitungsgebiet, im südlichen Brandenburg und in Nordwestsachsen, häufig Rükkenmesserchen mit retuschiertem Ende als Elemente des mitteldeutschen Spätmagdaléniens. $^{26}$ Letztere finden wir auch in Birkes Fundmaterial, so in Oelschütz und Dehnitz (»Wüste Kirche«). - Die hiesigen Fundplätze reihen sich entlang der Mulde auf deren Hochufer oder auf sanften Kuppen, die meisten von ihnen erfuhren in der Nacheiszeit eine abermalige Nutzung als Siedelplätze mittelsteinzeitlicher Standwildjäger, wie kulturspezifische mesolithische Geräte im Fundmaterial anzeigen (s. unten). Wichtigstes Jagdtier der Rückenspitzen-Leute scheint, der Besserung des Klimas in der Alleröd-Wärmeschwankung und der Ausbreitung lichter Wälder mit Birken und Kiefern entsprechend, der Hirsch gewesen zu sein. ${ }^{27}$

Die folgende Jüngere Dryaszeit als letztes Jahrtausend des Eiszeitalters war mit einem deutlichen Temperaturrückgang verbunden, die Klima- und Vegetationszonen verschoben sich nach Süden, und die Steppentundra brei- 
tete sich erneut aus. ${ }^{28}$ Als Folge dieses Kälterückschlages drangen die von spezialisierten Rentierjägern getragenen und archäologisch durch kleine gestielte Pfeilspitzen charakterisierten Stielspitzen-Gruppen ${ }^{29}$ aus dem Norden nach Brandenburg ${ }^{30}$ und in das ostelbische Nordsachsen vor, wo am Rande des Elbtales bei Nünchritz und in der Oberlausitz $^{31}$ die südlichsten Fundpunkte von Stielspitzen - mehrheitlich des Typs Ahrensburg - in Mitteldeutschland liegen. In Sachsen westlich der Elbe gibt es keine einzige Stielspitze. Dieses Gebiet gehörte seit der Ältesten Dryaszeit zum Siedlungsraum des mitteldeutschen Spätmagdaléniens zwischen Saale und oberer Elbe ${ }^{32}$ und weist in Nordwestsachsen, insbesondere entlang der Mulde, ausschließlich Fundstellen des mitteldeutschen Spätmagdaléniens und des Rückenspitzen-Kreises auf (s. oben). Das Fehlen von Stielspitzen - so auch in der Sammlung Birke - dürfte anzeigen, dass hier die Jäger der Rissener Gruppe ihre in der Allerödzeit in Besitz genommenen Schweifgebiete auch während der Jüngeren Dryaszeit besetzten; ob über das ganze Jahrtausend dieses Zeitabschnittes hinweg, ist offen und ohne geologisch-stratigrafisch oder anderweitig naturwissenschaftlich datierte Funde und Befunde nicht sicher $\mathrm{zu}$ bestimmen. Die hinter den verschiedenen archäologischen Formengruppen stehenden Gemeinschaften hielten sich demnach trotz der Lebensweise als schweifende Jäger über Jahrhunderte hinweg an bestimmte Räume und respektierten die der Nachbargemeinschaften. $^{33}$ - Rückenspitzen- und Stielspitzen-Gruppen bilden den jüngsten Abschnitt der Altsteinzeit, das Spätpaläolithikum (Späte Altsteinzeit).

Das Ende der Jüngeren Dryaszeit um 9.600 v. $\mathrm{Chr}^{34}$ bezeichnet zugleich das Ende der letzten Eiszeit. Klima und Vegetation - und damit verbunden die Tierwelt - wandelten sich grundlegend. In einem Zuge mit der allmählichen Erwärmung breitete sich dauerhaft der Wald aus, im Präboreal als dem ersten vegetationsgeschichtlichen Abschnitt der Nacheiszeit waren es Birken-, später Kiefernwälder, denen im Boreal die Hasel und in der Spätphase der Eichenmischwald folgten, aus welchem schließlich der Eichenmischwald des Atlantikums hervorging. ${ }^{35}$ Mit dem Wald hielt typisches Standwild wie Elch, Rothirsch, Reh und Wildschwein Einzug. Die archäologischen Kulturen der Jäger, Sammler und Fischer der Zeitspanne zwischen dem Ende der letzten Eiszeit um 9.600 v. Chr. und dem Beginn der Landnahme durch bäuerliche

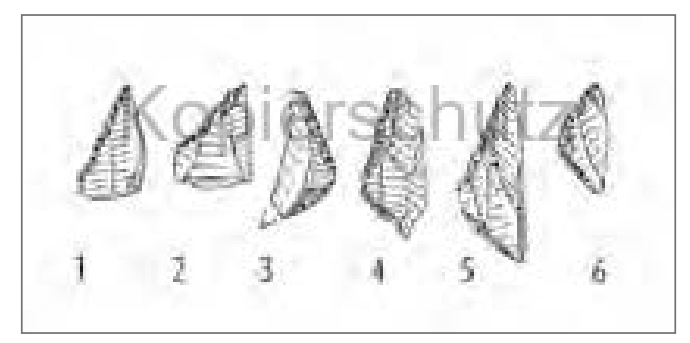

Nemt: Geometrische

Mikrolithen (1-6). 2:3.

(aus: V. Geupel 1985 [Anm. 3] Taf. 64, 6-11).

(c) Landesamt für

Archäologie Sachsen

Bevölkerungsgruppen um 5.500 v. Chr. werden als Mesolithikum (Mittlere Steinzeit) zusammengefasst. ${ }^{36}$ Aus den schweifenden Jägern der Späteiszeit, die mit dem Wild der Tundra über weite Strecken hin- und hergependelt waren, wurden, den neuen Tierarten entsprechend, Standwildjäger. Durch die Vegetationsverhältnisse waren aber Beweglichkeit und Kommunikation der Bevölkerungsgruppen untereinander eingeschränkt, was mit einer temporären Sesshaftigkeit einherging. ${ }^{37}$ Der Mensch bewegte sich nun in einem begrenzten Raum, Nahrungs- und Rohstofferwerb erfolgten aber nach wie vor in "Aneignungswirtschaft» aus der vorgefundenen, natürlichen Umwelt. ${ }^{38}$ Auf Grund ihrer Unvergänglichkeit bilden Steinwerkzeuge, wie in den vorausgegangenen Perioden vor allem aus Feuerstein (Flint) hergestellt, auch für die Mittlere Steinzeit das wichtigste Fundmaterial. Sehr charakteristisch sind sog. geometrische Mikrolithen (»kleine Steine", von griech.: mikros = klein, lithos = Stein), welche, wie Funde aus Mooren lehren, als Einsatzklingen - beispielsweise als Widerhaken und Schneiden an Pfeilen - verwendet worden waren. Sie sind bereits aus der Späten Altsteinzeit bekannt, wo sie zum Steingerätinventar der Ahrensburger Stielspitzen-Gruppe gehörten $^{39}$, Anzahl und Formenvielfalt sind aber vor allem für die Mittlere Steinzeit kennzeichnend. Eine Neuentwicklung stellten Steinbeile dar. Die aus Feuerstein gefertigten Beile werden nach Herstellungstechnik und Funktion in Kern- und Scheibenbeile geschieden: Schäftungen, die bei günstiger Feuchtbodenlagerung erhalten geblieben sind, zeigen, dass das Kernbeil tatsächlich als Beil benutzt wurde, während für das Scheibenbeil, für welches entsprechende Handhaben bislang fehlen, angenommen wird, dass es als Dechsel fungierte. Beide Feuerstein-Großgeräte sind in den Fundinventaren der Sammlung Birke repräsentativ vertreten und schließen das hiesige Mesolithikum dem nordmitteleuropäisch-südskandinavischen Kern- und Scheibenbeil-Kreis an, dessen Verbreitungsgebiet von der westlichen Ostsee über Brandenburg und das Mittelelbgebiet bis nach Sachsen
Pfeilfragment mit eingesetzten Mikrolithen aus dem Lilla Loshult-Moor (Schweden).

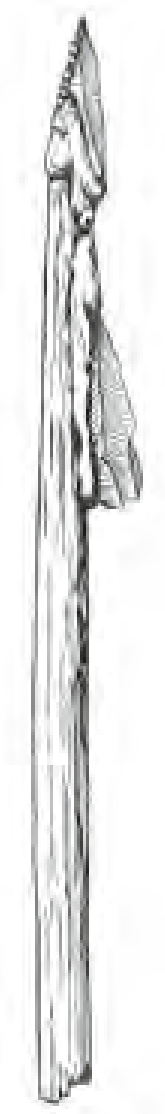



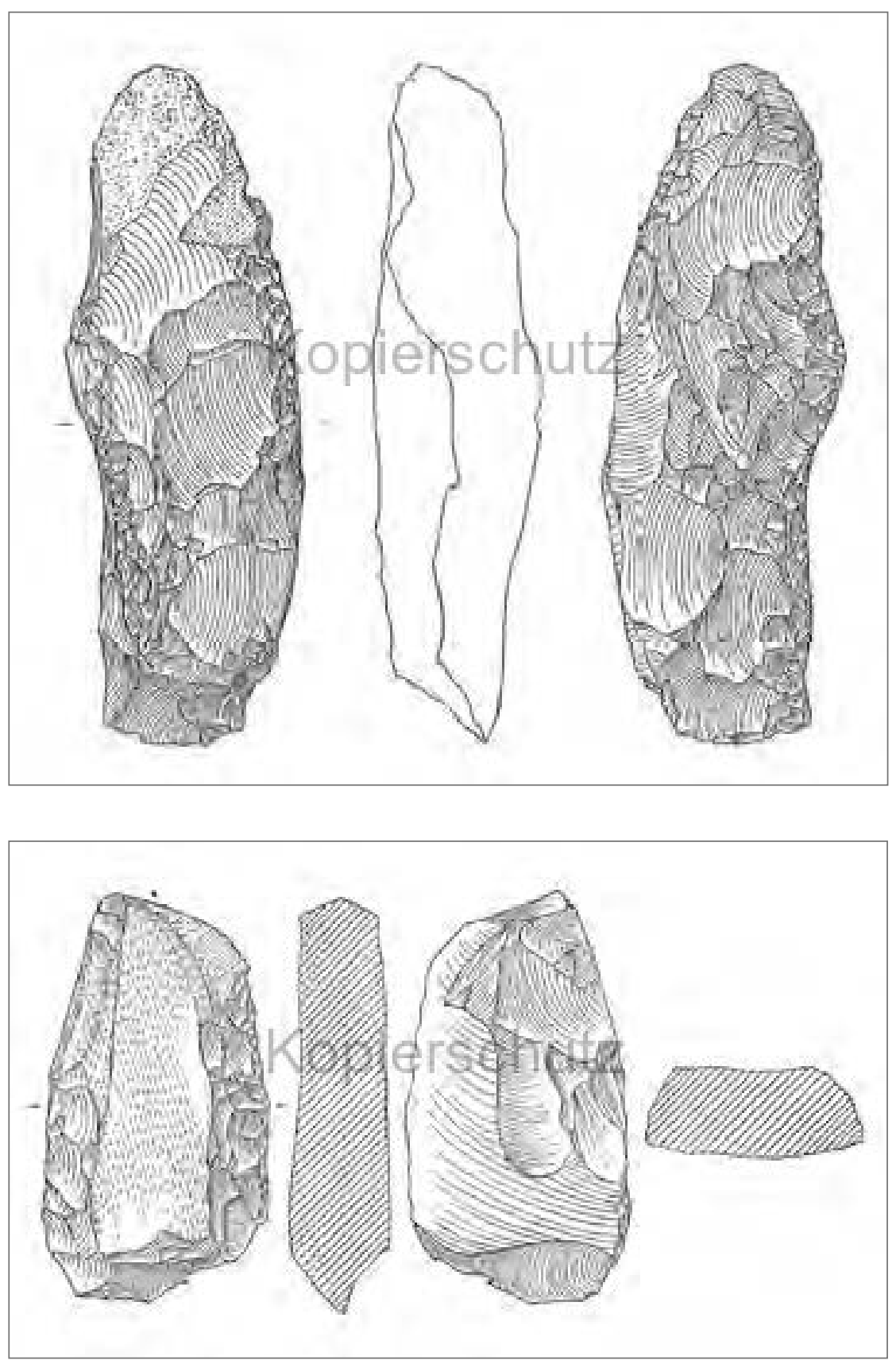

Oelschütz: Kernbeil. 2:3. (aus: V. Geupel 1985 [Anm. 3], Taf. 72, 2). (O) Landesamt für Archäologie Sachsen

Walzig: Scheibenbeil. 2:3. (aus: V. Geupel 1985 [Anm. 3], Taf. 85, A). @ L Landesamt für Archäologie Sachsen

\section{Autor \\ Volkmar Geupel Dresden}

reichte. ${ }^{40}$ Wie oben erwähnt, waren das Hochufer und kleinere Kuppen über der Mulde auch in der Mittelsteinzeit bevorzugte Siedlungsplätze, wie die typischen Feuersteinwerkzeuge beweisen, die R. Birke vor allem in Dehnitz, Nemt, Oelschütz (4 dicht benachbarte Fundstellen), Pausitz (2 Plätze) und Walzig (2 Plätze) $)^{41}$ bergen konnte; von den drei letztgenannten Fundorten liegen klassische Kern- und Scheibenbeile vor. Die für diese steinzeitliche Periode ebenfalls charakteristischen Geräte aus Felsgestein (Walzenbeile, durchlochte Hauen, Keulenköpfe) sind bislang im Wurzener Land - wohl rein zufällig - noch nicht gefunden worden, und für die Überlieferung von Werkzeugen und anderen Gegenständen aus organischem Material wie Knochen, Geweih, Holz, Rinde und Bast, die von Fundstellen mit Feuchtböden im nördlichen Mitteleuropa gut bekannt sind, fehlten hier die konservierenden natürlichen Bedingungen. - Die Ernährungsgrundlage der mittelsteinzeitlichen Bevölkerung bildete die kombiniert betriebene Jagd- und Sammelwirtschaft, neben die mit größerer Bedeutung als in der Altsteinzeit der Fischfang trat. Von dem waldbewohnenden Standwild spielten als Jagdtiere offenbar Hirsch und Wildschwein eine Rolle. ${ }^{42}$ Das Ende des Mesolithikums als Periode der letzten Jäger, Sammler und Fischer in unserem Raume um 5.500 v. Chr. war mit einem signifikanten Umbruch der Wirtschaftsform, nämlich der Durchsetzung von Ackerbau und Viehzucht, verbunden, und der Mensch war auf Dauer sesshaft geworden. In der archäologischen Periodisierung wird die Zeit zwischen um 5.500 und um 2.200 v. Chr. ${ }^{43}$ als Neolithikum (Jüngere Steinzeit) bezeichnet. Die einwandernden Bauern der frühen Jungsteinzeit (um 5.500 - um 4.500 v. $\mathrm{Chr}^{4}{ }^{44}$ ) siedelten sich auf den fruchtbaren Böden des Leipziger Landes, des mittelsächsischen Lößhügellandes zwischen Grimma und Meißen sowie der Dresdener Elbtalweitung an, wovon dort die große Dichte ihrer archäologischen Hinterlassenschaften zeugt. ${ }^{45}$ Wurzen und sein weiteres Umland blieben davon unberührt.

Damit sei die Skizze über die früheste Besiedlung des Muldelandes um Wurzen abgeschlossen. Die Grundlage dafür schuf der Heimatforscher R. Birke, dessen planmäßigem Absuchen des Geländes nach archäologischen Gegenständen die Kenntnis des hiesigen steinzeitlichen Fundraumes verdankt wird. $25 \mathrm{~km}$ muldeaufwärts erschloss W. C. Pfau (1862-1946) bereits vor dem Ersten Weltkrieg einen solchen um Rochlitz, dessen zeitlich-kulturelles Fundspektrum nahezu identisch ist. ${ }^{46}$ Von dem Flussabschnitt zwischen beiden Fundräumen liegen, da es hier vergleichbare Sammlerinitiativen nicht gegeben hat, nur wenige, aber charakteristische endpaläolithische und mesolithische Funde vor. ${ }^{47}$ Sie bilden die Brücke zwischen den zwei Sammelzentren und verbinden diese zu einer von der Mulde bestimmten Fundlandschaft, die einen Einblick in die Besiedlung und Nutzung eines Flussbereiches durch schweifende Jäger und Sammler des späten Eiszeit- und des Nacheiszeitalters gewährt. 


\section{Anmerkungen}

1 R. Birke, Altsteinzeitliche Funde aus Groitzsch, Kr. Eilenburg. Ein Vorbericht. In: Arbeits- u. Forschungsberichte z. sächsischen Bodendenkmalpflege 4, 1954, S. 21-30.

2 H. Hanitzsch, Groitzsch bei Eilenburg. Schlag- und Siedlungsplätze der späten Altsteinzeit. Veröff. d. Landesmuseums f. Vorgeschichte Dresden, Bd. 13, Berlin 1972. - Kleinere, durch Baumaßnahmen verursachte Grabungen erfolgten durch das Landesamt für Archäologie 1996 und 2004 (M Seiler et al., Ein neuer Schlagplatz des Spätmagdalénien von Groitzsch bei Eilenburg [Lkr. Delitzsch]. In: Arbeits- u. Forschungsberichte z. sächsischen Bodendenkmalpflege 41, 1999, S. 17-25; I. Kraft, Steinzeitjägern auf der Spur. Neues vom Kapellenberg in Groitzsch bei Eilenburg. In: Archæo 2, 2005, S. 10f.)

3 V. Geupel, Spätpaläolithikum und Mesolithikum im Süden der DDR. Katalog Teil 1, Bezirke Dresden, Karl-Marx-Stadt und Leipzig. Veröff. d. Landesmuseums f. Vorgeschichte Dresden, Bd. 17, Berlin 1985, S. 25-35 u. Taf. 58 A/B u. 64-102 B.

4 Die Weichseleiszeit endete mit der Jüngeren Dryas- oder Tundrazeit exakt 11.590 vor heute (2000) (T. Litt et al., Stratigraphische Begriffe für das Quartär des norddeutschen Vereisungsgebietes. In: Eiszeitalter u. Gegenwart, Quarternary Science Journal, Vol. 56, No. 1/2, 2007, S. 7-65, hier: S. 63).

5 K. Pietzsch, Abriss der Geologie von Sachsen, Berlin 1951, S. $145 \mathrm{f}$.

6 V. Geupel, Altsteinzeitliche Feuersteingeräte vom „Spitzberg“ bei Lüptitz, Kr. Wurzen. In: Ausgrabungen u. Funde $17,1972,51-58$.

7 Geologische Specialkarte des Königreichs Sachsen, No. 13 Section Wurzen - Altenbach (Geolog. Revision z. 2. Auflage 1902).

8 Messtischblatt 13, Section Wurzen, Ausgabe 1879.

9 G. Haase, Nordsächsisches Platten- und Hügelland In: K. Mansfeld/H. Richter (Hrsg.), Naturräume in Sachsen. Forschungen z. Deutschen Landeskunde Bd. 238, Trier 1995, S 87-92, hier: S. $88 \mathrm{f}$.

10 V. Toepfer/D. Mania, Paläolithische Funde vom Petersberg nördlich von Halle (Saale). In: Jahresschrift f. mitteldeutsche Vorgeschichte 57, 1973, S. 49-69.

11 K. Pietzsch (Anm. 5), S. 146

12 13.800-11.590 vor heute (2000) (T. Litt et al. [Anm. 4], S. 61-64).

13 G. Bosinski, Paläolithikum und Mesolithikum. In: P. Woldstedt/K. Duphorn, Norddeutschland und angrenzende Gebiete im Eiszeitalter, Stuttgart 1974, S. 1-20 Paläolithikum, S. 21-24 Mesolithikum, hier: S. 15 f.; H. Hanitzsch 1972 (Anm. 2), S. 115 (Chronologie-Tabelle des mitteldeutschen Spätmagdaléniens).

14 H. Hanitzsch 1972 (Anm. 2), S. 97-99 und Taf. 76, 5; 77; I. Kraft, Some remarks on an old work of art. The horse engravings from Groitzsch, Saxony, Germany. Anthropologie XLVII/1-2, 2009, S. 153-158.

15 V. Geupel, Zwei spätpaläolithische Fundplätze im Wurzener Land. In: Arbeits- u. Forschungsberichte z. sächsischen Bodendenkmalpflege 16/17, 1967, S. 7-25.

16 V. Toepfer, Stratigraphie und Ökologie des Paläolithikums In: Periglazial-Löß-Paläolithikum im Jungpleistozän der Deutschen Demokratischen Republik (Erg.-Heft 274 zu Petermanns Geograph. Mitteilungen), Gotha/Leipzig 1970 S. 329-422, hier: S. 409 f.; H. Hanitzsch 1972 (Anm. 2), S 106-114 u. Abb. 22

17 G. Bosinski (Anm. 13), S. 12

18 Ebenda, S. 16

19 13.350-12.680 vor heute (2000) (T. Litt et al. [Anm. 4], S. 62 f.)

20 H. Schwabedissen, Das Alter der Federmesser-Zivilisation auf Grund neuer naturwissenschaftlicher Untersuchungen. Mit Beiträgen v. R. Schütrumpf u. K. O. Münnich. In: Eiszeitalter u. Gegenwart 8, 1957, S. 200-209.

21 Ders., Die Federmesser-Gruppen des nordwesteuropäi- schen Flachlandes. Zur Ausbreitung des Spät-Magdalénien. Neumünster 1954, S. 8 f. u. Abb. 11 a-l, Abb. 12 f-v; W. Taute, Paläolithikum. Histor. Handatlas v. Brandenburg u. Berlin, Nachträge H. 7 (Veröff. d. Histor. Kommission zu Berlin), Berlin 1980, S. 10, Abb. 3-4.

22 G. Bosinski (Anm. 13), S. 17

23 Zum Fundmaterial von den genannten Fundplätzen s. V. Geupel 1985 (Anm. 3).

24 W. Taute, Funde der spätpaläolithischen „Federmessergruppen“ aus dem Raum zwischen mittlerer Elbe und Weichsel. In: Berliner Jahrbuch f. Vor- u. Frühgeschichte 3, 1963, S 62-111, hier: S. 99; Ders. 1980 (Anm. 21), S. 3; G. Bosinski (Anm. 13), S. 17.

25 H. Schwabedissen 1954 (Anm. 21), Taf. 102 b; W. Taute 1963 (Anm. 24), S. 99; Ders. 1980 (Anm. 21), S. 5.

26 H. Schwabedissen 1954 (Anm. 21), S. 64 f., 74; W. Taute 1963 (Anm. 24), S. 99; G. Bosinski (Anm. 13), S. 18.

27 G. Bosinski (Anm. 13), S. 18.

28 12.680-11.590 vor heute (2000) (Litt et al. [Anm. 4], S. 63 f. u. Abb. 6).

29 W. Taute, Die Stielspitzen-Gruppen im nördlichen Mitteleuropa. Ein Beitrag zur Kenntnis der späten Altsteinzeit (Fundamenta, Monographien zur Urgeschichte, hrsg. v. H. Schwabedissen, Reihe A, Bd. 5), Köln Graz 1968.

30 Ders. 1980 (Anm. 21), S. 4 f.

31 V. Geupel 1985 (Anm. 3), S. 9, 12f., 14; I. Kraft, Paläolithikum und Mesolithikum. In: R. Heynowski/R. Reiß (Red.), Atlas z. Geschichte u. Landeskunde v. Sachsen, Beiheft z. Karte B I, 1. 1-15. Leipzig u. Dresden 2010, S. 18 u. Karte B I 1.1.

32 W. Taute 1968 (Anm. 29), S. 265 u. Abb. 57; Ders. 1980 (Anm. 21), S. 6 f.; H. Hanitzsch 1972 (Anm. 2), S. 108 u. Abb. 21.

33 W. Taute 1968 (Anm. 29), S. 265; Ders. 1980 (Anm. 21), S. 7.

34 Wie Anm. 4

35 D. Mania, Das Eiszeitalter zwischen Thüringer Wald und mittlerer Elbe. II. Erdgeschichtlicher Ablauf. In: Urgeschichte u. Heimatforschung 11, 1973, Tab. 12 (S. 70).

36 Archäologisch wird zwischen älterem Mesolithikum in Präboreal und Boreal und jüngerem Mesolithikum im Atlantikum unterschieden, was aber hier vernachlässigt werden kann.

37 G. Bosinski (Anm. 13), S. 21.

38 H. Schwabedissen, Sinngehalt und Abgrenzung des Mesolithikums nach den Forschungsergebnissen im nördlichen Teil des europäischen Kontinents. In: Report of the VIth Intern. Congr. on Quarternary Warschau 1961, S. 383-401.

39 W. Taute 1968 (Anm. 29), S. 214

$40 \mathrm{H}$. Schwabedissen, Die mittlere Steinzeit im westlichen Norddeutschland. Neumünster 1944, S. 119-129; V. Geupel, Zur Kenntnis des Mesolithikums im Süden der DDR. In: S. K. Kozłowski (Hrsg.), The Mesolithic in Europe, Warsaw 1973, S. 157-176.

41 Zum Fundmaterial von den genannten Fundstellen s. V. Geupel 1985 (Anm. 3).

42 G. Bosinski (Anm. 13), S. 22.

43 H. Stäuble et al., Neolithikum. In: R. Heynowski/R. Reiß (Red.), Atlas z. Geschichte u. Landeskunde v. Sachsen, Beiheft z. Karte B I 1.1-1.5. Leipzig u. Dresden 2010, S. 24-73.

44 H. Stäuble, Frühneolithikum. In: Ebenda, S. 24-42, hier S. 25 u. Karte B I 1.2

45 Ebenda, S. 27-31 u. Karte B I 1.2.

46 H. Hanitzsch, Die steinzeitlichen Funde im Heimatmuseum Rochlitz (Ein Vorbericht). In: Leipziger Beiträge z. Vor- u. Frühgeschichte (Forschungen z. Vor- u. Frühgeschichte Nr. 1), Leipzig 1955, S. 15-19; V. Geupel 1985 (Anm. 3), S. 15-23 (Kreis Rochlitz); I. Kraft 2010 (Anm. 31), S. 20 f. u. Karte B I 1.1.

47 V. Geupel 1985 (Anm. 3), S. 25 f. (Kreis Grimma). 